Research Article

\title{
Best Lag Window for Spectrum Estimation of Law Order MA Process
}

\author{
Ali Sami Rashid $\left(\mathbb{D}\right.$, Mohammed Jabber Hawas Allami $\mathbb{D}^{D}$, and Ahmed Kareem Mutasher \\ Department of Mathematics, College of Education, Misan University, Amarah, Iraq \\ Correspondence should be addressed to Ali Sami Rashid; alisamirashid@uomisan.edu.iq
}

Received 9 August 2019; Accepted 6 February 2020; Published 17 March 2020

Academic Editor: Beong In Yun

Copyright (c) 2020 Ali Sami Rashid et al. This is an open access article distributed under the Creative Commons Attribution License, which permits unrestricted use, distribution, and reproduction in any medium, provided the original work is properly cited.

\begin{abstract}
In this article, we investigate spectrum estimation of law order moving average (MA) process. The main tool is the lag window which is one of the important components of the consistent form to estimate spectral density function (SDF). We show, based on a computer simulation, that the Blackman window is the best lag window to estimate the SDF of MA(1) and MA(2) at the most values of parameters $\beta_{i}$ and series sizes $n$, except for a special case when $\beta=-1$ and $n \geq 40$ in MA(1). In addition, the Hanning-Poisson window appears as the best to estimate the SDF of MA(2) when $\beta_{1}=\beta_{2}=-0.5$ and $n \geq 40$.
\end{abstract}

\section{Introduction}

A set $x_{t}$ of numerical data (observations) made sequentially in time $t$ is called time series [1]. There are some important processes of a time series: autoregressive, moving average, and autoregressive-moving average processes.

Spectral analysis can be defined as a process that assigns power versus frequency. One of the time series analysis techniques is spectral analysis. The object of spectral analysis is to estimate and study the spectrum of the time series processes for the phenomena of physics and engineering [2].

The spectrum estimation methods can be classified into parametric and nonparametric methods [3]. The consistent estimate of spectral density function $\widehat{f}(\omega)$ is the most important nonparametric spectral analysis method, which depends on lag window $\lambda_{T}(v)$ and truncation point $T$ [4].

Window functions are used in the estimation of power spectra and bispectra in order to ensure the consistency of the periodogram and the Fourier-type bispectrum estimation methods. A three-dimensional optimum bias lag window is introduced in the estimation of the 4th-order cumulant spectrum, also called trispectrum, which is estimated from the threedimensional Fourier transform of the 4th order cumulants [5].

Zhongsheng et al. [6] suggested that using windows is one important way to improve bispectrum estimation and also an appropriate window function can be used to reduce variance and suppress noise, but it was noticed that sidelodes in a spectrum of window functions can be ended up in spectrum leak. Thus, one urgent problem which needed to be solved for the application of bispectrum was how to find one appropriate window. He combined a new lag window with Hanning-Poisson window without sidelodes, which is used for nonparametric bispectrum estimation instead of rectangle window. When the spatial location area increases becoming extremely large, it is very difficult [7], or not possible, to evaluate the covariance matrix determined by the set of location distance even for gridded stationary Gaussian process. To alleviate the numerical challenges, he did construct a nonparametric estimator called periodogram of spatial version to represent the sample property in the frequency domain because periodogram requires less computational operation by fast Fourier transform algorithm. Under some regularity conditions on the process, he investigated the asymptotic unbiasedness property of the periodogram as estimator of the spectral density function and achieved the convergence rate.

The basic concepts given in Sections 2-5 present white noise, moving average process of order $q$ and their properties, spectral density function (SDF) on general and SDF of MA(q), and the consistent estimate of SDF, and some 
important lag windows are reviewed. Section 6 presents a simulation for comparison between the SDF and the consistent estimate of SDF.

\section{White Noise}

A purely random process $\epsilon_{t}, t \in \mathbb{Z}^{+}$is called white noise (Gaussian noise) if it consists of a sequence of uncorrelated independent identically distributed (i.i.d) random variables [3], with mean $\mu_{\epsilon}=0$, variance $\operatorname{var}\left(\epsilon_{t}\right)=\sigma_{\epsilon}^{2}$, and the autocovariance function

$$
R(v)=\operatorname{cov}\left(\epsilon_{t}, \epsilon_{t+v}\right)= \begin{cases}\sigma_{\epsilon}^{2}, & v=0 \\ 0, & v \neq 0\end{cases}
$$

In addition, the autocorrelation function $\rho_{v}$ is

$$
\rho_{v}=\frac{R(v)}{R(0)}=\frac{E\left(x_{t} x_{t+v}\right)}{\sigma_{x}^{2}}= \begin{cases}1, & |v| \leq q \\ 0, & |v|>q .\end{cases}
$$

\section{Moving Average Process}

A stochastic process $x_{t}, t \in \mathbb{Z}^{+}$is called moving average process of order $q$ and denoted by $\operatorname{MA}(q)$. This is given by

$$
x_{t}=\sum_{i=0}^{q} \beta_{i} \epsilon_{t-i}
$$

where $\epsilon_{t}$ is the white noise with mean zero and covariance $\sigma_{\epsilon}^{2}$ and $\beta_{i} . \beta_{i}=0, i>q$ is the coefficient of the process. The statistical properties of $\operatorname{MA}(q)$ is

$$
\begin{aligned}
& \mu_{x}=E\left(x_{t}\right)=\sum_{i=0}^{q} \beta_{i} E\left(\epsilon_{t-i}\right)=0, \\
& \sigma_{x}^{2}=\operatorname{var}\left(x_{t}\right)=\sum_{i=0}^{q} \beta_{i}^{2} \operatorname{var}\left(\epsilon_{t-i}\right)=\sigma_{\epsilon}^{2}\left(\sum_{i=0}^{q} \beta_{i}^{2}\right),
\end{aligned}
$$

such that $\epsilon_{t}$ be the uncorrelated random process. As a result, the autocovariance function $R(v)$ cuts off after a point $x_{t}, t>q$, and that implies $\operatorname{cov}\left(x_{t}, x_{t+v}\right),|v|>q$ and $R(v)$ as

$$
R(v)=E\left(x_{t} x_{t+v}\right)= \begin{cases}\sigma_{\epsilon}^{2}\left(\sum_{i=0}^{q} \beta_{i} \beta_{i+v}\right), & |v| \leq q, \\ 0, & |v|>q .\end{cases}
$$

The autocorrelation function

$$
\rho_{v}=\frac{R(v)}{R(0)}=\frac{E\left(x_{t} x_{t+v}\right)}{\sigma_{x}^{2}}= \begin{cases}\frac{\sigma_{\epsilon}^{2}\left(\sum_{i=0}^{q} \beta_{i} \beta_{i+v}\right)}{\sigma_{x}^{2}}, & |v| \leq q, \\ 0, & |v|>q .\end{cases}
$$

Note that $\mu_{x}, \sigma_{x}^{2}, R(v)$, and $\rho_{v}$ are constants, the finite does not depend on time $t$ for any finite order $q$. Thus, the moving average process $\mathrm{MA}(q)$ of finite order $q$ is a stationary process [2]. As a special case, MA(1),

$$
x_{t}=\beta_{0} \epsilon_{t}+\beta_{1} \epsilon_{t-1} \text {. }
$$

And $\mu_{x}=0, \sigma_{x}^{2}=\operatorname{var}\left(x_{t}\right)=\sigma_{\epsilon}^{2}\left(\beta_{0}^{2}+\beta_{1}^{2}\right)$, and the autocovariance and autocorrelation functions are given by

$$
\begin{aligned}
& R(v)= \begin{cases}\sigma_{\epsilon}^{2}\left(\beta_{0} \beta_{v}+\beta_{1} \beta_{1+v}\right), & |v| \leq 1, \\
0, & |v|>1,\end{cases} \\
& \rho_{v}= \begin{cases}\frac{\sigma_{\epsilon}^{2}\left(\beta_{0} \beta_{v}+\beta_{1} \beta_{1+v}\right)}{\sigma_{x}^{2}}, & |v| \leq 1, \\
0, & |v|>1 .\end{cases}
\end{aligned}
$$

So, MA(2) is defined as

$$
x_{t}=\beta_{0} \epsilon_{t}+\beta_{1} \epsilon_{t-1}+\beta_{2} \epsilon_{t-2} \text {. }
$$

And, the expected value $\mu_{x}=E\left(\beta_{0} \epsilon_{t}+\beta_{1} \epsilon_{t-1}+\beta_{2} \epsilon_{t-2}\right)=0$, and the variance is

$$
\sigma_{x}^{2}=\sigma_{\epsilon}^{2}\left(\beta_{0}^{2}+\beta_{1}^{2}+\beta_{2}^{2}\right) \text {. }
$$

It is clear that $\sigma_{x}^{2}=R(v=0)$, and the autocovariance and autocorrelation functions are given by

$$
\begin{aligned}
& R(v)= \begin{cases}\sigma_{\epsilon}^{2}\left(\beta_{0} \beta_{v}+\beta_{1} \beta_{1+v}+\beta_{2} \beta_{2+v}\right), & |v| \leq 1, \\
0, & |v|>1,\end{cases} \\
& \rho_{v}= \begin{cases}\frac{\sigma_{\epsilon}^{2}\left(\beta_{0} \beta_{v}+\beta_{1} \beta_{1+v}+\beta_{2} \beta_{2+v}\right)}{\sigma_{x}^{2}}, & |v| \leq 1, \\
0 b, & |v|>1 .\end{cases}
\end{aligned}
$$

\section{Spectral Density Function}

If $x_{t}, t \in \mathbb{Z}$ is a discrete stochastic process with autocorrelation function $\rho_{v}[3,8]$, a spectral density function (SDF) $f(\omega)$ is defined as a Fourier transform of autocorrelation function $\rho_{v}$ and is given as

$$
f(\omega)=\frac{1}{2 \pi} \sum_{v=-\infty}^{\infty} \rho_{\nu} e^{-j \omega v}
$$

where $j=\sqrt{-1}$. The formula is rewritten as

$$
f(\omega)=\frac{1}{2 \pi}\left[\sum_{v=-\infty}^{-1} \rho_{v} e^{-j \omega v}+\rho_{0}+\sum_{v=1}^{\infty} \rho_{v} e^{-j \omega v}\right],
$$

$$
f(\omega)=\frac{1}{2 \pi}\left[\sum_{v=1}^{\infty} \rho_{-v} e^{j \omega v}+\rho_{0}+\sum_{v=1}^{\infty} \rho_{\nu} e^{-j \omega v}\right] .
$$

Since autocorrelation is an even function [9], it implies $\rho_{v}=\rho_{-v}$ and $\rho_{0}=1$. Thus,

$$
f(\omega)=\frac{1}{2 \pi}\left[1+\sum_{v=1}^{\infty} \rho_{\nu}\left(e^{2 \pi j \omega v}+e^{-2 \pi j \omega v}\right)\right] .
$$


Hence,

$$
f(\omega)=\frac{1}{2 \pi}\left[1+2 \sum_{v=1}^{\infty} \rho_{v} \cos (\omega v)\right]
$$

4.1. SDF of $M A(q)$. Let $x_{t}$ be the moving average process defined in (3) with autocovariance function $R(v)$ and autocorrelation function $\rho_{v}$. The spectral density function $f(\omega)$ defined in (13) is given as

$$
\begin{aligned}
f(\omega) & =\frac{1}{2 \pi} \sum_{v=-\infty}^{\infty} \rho_{v} e^{-j \omega v} \\
& =\frac{1}{2 \pi}\left[\sum_{v=-\infty}^{-(q+1)} \rho_{\nu} e^{-j \omega v}+\sum_{v=-q}^{q} \rho_{\nu} e^{-j \omega v}+\sum_{v=q+1}^{\infty} \rho_{\nu} e^{-j \omega v}\right] .
\end{aligned}
$$

From (7),

$$
\rho_{v}= \begin{cases}\frac{\sigma_{\epsilon}^{2}\left(\sum_{i=0}^{q} \beta_{i} \beta_{i+v}\right)}{\sigma_{x}^{2}}, & |v| \leq q, \\ 0, & |v|>q .\end{cases}
$$

Then,

$f(\omega)=\frac{1}{2 \pi}\left[\sum_{v=-q}^{-1} \rho_{\nu} e^{-j \omega v}+\left.\rho_{\nu} e^{-j \omega v}\right|_{v=0}+\sum_{v=0}^{q} \rho_{\nu} e^{-j \omega v}\right]$.

Since $\rho_{v}=\rho_{-v}$ and $\rho_{v}=1$,

$$
\begin{aligned}
f(\omega) & =\frac{1}{2 \pi}\left[1+\sum_{v=1}^{q} \rho_{v}\left(e^{j \omega v}+e^{-j \omega v}\right)\right] \\
& =\frac{1}{2 \pi}\left[1+2 \sum_{v=1}^{q} \rho_{v} \cos (\omega v)\right] .
\end{aligned}
$$

Hence,

$$
f(\omega)=\frac{1}{2 \pi}\left[1+\sum_{v=1}^{q} \frac{\sigma_{\epsilon}^{2}\left(\sum_{i=0}^{q} \beta_{i} \beta_{i+v}\right)}{\sigma_{x}^{2}} \cos \omega v\right] .
$$

As a special case, MA(1), the spectral density function will be

$$
f(\omega)=\frac{1}{2 \pi}\left[1+\frac{\sigma_{\epsilon}^{2}\left(\beta_{0} \beta_{1}\right)}{\sigma_{x}^{2}} \cos \omega v\right] .
$$

And spectral density function of MA(2) is given by

$$
f(\omega)=\frac{1}{2 \pi}\left[1+\sum_{v=1}^{2} \frac{\sigma_{\epsilon}^{2}\left(\beta_{0} \beta_{v}+\beta_{1} \beta_{1+v}+\beta_{2} \beta_{2+v}\right)}{\sigma_{x}^{2}} \cos \omega v\right] .
$$

\section{The Consistent Estimate of SDF}

Let $X_{t}, t \in \mathbb{Z}$ be a real-valued, weakly stationary, discrete stochastic process (time series) with zero mean and autocovariance function $R_{v}$ with lag $v$ and autocorrelation function $\rho_{v}$ [3]. The consistent estimate of $R_{v}$ and $\rho_{v}$ are

$$
\begin{aligned}
& \widehat{R}_{v}=\frac{1}{n} \sum_{t=1}^{n-|v|} X_{t} X_{t+|v|}, \quad v<n, \\
& \widehat{\rho}_{v}=\frac{\widehat{R}_{v}}{\widehat{R}_{0}}=\frac{\sum_{t=1}^{n-|v|} X_{t} X_{t+|v|}}{\sum_{t=1}^{n} x_{t}^{2}} .
\end{aligned}
$$

If $X_{t}$ is a stochastic process of size $n$, then the consistent form to estimate the spectral density function is [2]

$$
\widehat{f}(\omega)=\frac{1}{2 \pi} \sum_{v=-T+1}^{T-1} \hat{\rho}_{v} \lambda_{T}(v) \cos (v \omega), \quad-\pi \leq \omega \leq \pi,
$$

where $T$ is the truncation point $0 \leq T \leq n$ and $\lambda_{T}(v)$ is the lag window, which weighting the autocorrelation function.

The consistent estimate of SDF depends on two important sides, select an appropriate value of a truncation point $T$ and an appropriate lag window $\lambda_{T}(v)$.

There are a lot of lag windows suggested by researchers $[3,6,10-12]$. Table 1 contains the most important of lag windows as shown in previous papers.

\section{The Empirical Aspect}

A simulation experiment is applied to achieve our goal by using Matlab software according to the following assumptions:

(1) Generate $\mathrm{MA}(1) \quad$ process, $\quad x_{t}=\epsilon_{t}+\beta \epsilon_{t-1}$ and MA (2), $x_{t}=\epsilon_{t}+\beta_{1} \epsilon_{t-1}+\beta_{2} \epsilon_{t-2}$, where the white noise $\epsilon_{t}$ with $\mu_{\epsilon}=0$ and $\sigma_{\epsilon}^{2}=1$. Empirically, the initial white noise $\epsilon_{0}=\mu_{\epsilon}$ for $\mathrm{MA}(1)$ and $\epsilon_{0}=\mu_{\epsilon}$ and $\epsilon_{1}=\mu_{\epsilon}$ for MA(2). The parameter $\beta_{0}=1$ for all processes, with different values of the parameters $\beta, \beta_{1}$, and $\beta_{2}$ given in Tables 2 and 3.

(2) The different values of series sizes $n=10,100$, 500,1000 , and 10000 .

(3) The run size value of simulation $k=1000$.

(4) The appropriate value of a truncation point $T$ was calculated according to the closing window algorithm.

(5) The values of $\omega$ are $[-\pi:(0.0251): \pi]$ where the number of values is $L=250$, and $\hat{\rho}_{v}$ is defined in equation (25), and the lag windows $\lambda_{T}(v)$ are defined in Table 1.

(6) The spectral density function of moving average process $f\left(\omega_{i}\right)$ of MA (1) process is

$$
f(\omega)=\frac{1+2 \beta \cos \omega+\beta^{2}}{2 \pi\left(1+\beta^{2}\right)}, \quad-\pi \leq \omega \leq \pi,
$$




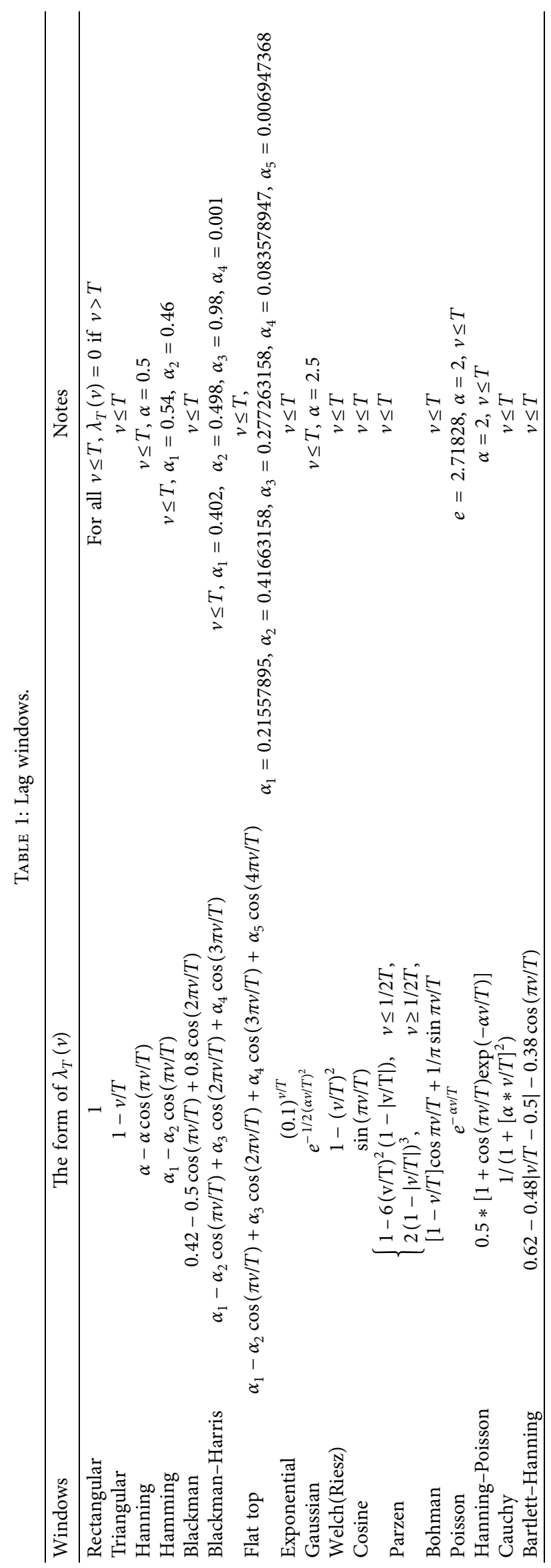


TABLE 2: Values of the parameter of MA(1).

\begin{tabular}{lcc}
\hline The value of $\beta$ & Different values of parameter $\beta$ \\
\hline
\end{tabular}

TABLE 3: Values of the parameters of MA(2).

\begin{tabular}{lccccccc}
\hline$\beta_{1}, \beta_{2}$ & \multicolumn{7}{c}{ Different values of parameters } \\
\hline$\beta_{1}$ & 1 & -1 & 0.5 & -0.5 & 0.5 & 0.5 & 1 \\
$\beta_{2}$ & -1 & 1 & 0.5 & -0.5 & -0.5 & 1 & -1.5 \\
\hline
\end{tabular}

TABLE 4: MSE values of MA(1) spectrum estimation with size $(n=10)$.

\begin{tabular}{|c|c|c|c|c|c|c|c|c|}
\hline \multirow{2}{*}{ Windows } & \multicolumn{8}{|c|}{ Different values of parameter $\beta$} \\
\hline & $\beta=-1.5$ & $\beta=-1$ & $\beta=-0.5$ & $\beta=0$ & $\beta=0.5$ & $\beta=1$ & $\beta=1.5$ & $\beta=2$ \\
\hline Rectangular & 6.317333361 & 6.316064139 & 6.319364383 & 6.332573978 & 6.345797365 & 6.349105366 & 6.347832956 & 6.345797365 \\
\hline Triangular & .311369594 & 6.310767548 & 6.31289241 & 6.321968603 & 6.329353747 & 6.331318921 & 6.330993772 & 6.330034865 \\
\hline Hanning & 6.32083044 & 6.318996447 & 6.323517904 & 6.341436557 & 6.360466439 & 6.36544573 & 6.363049374 & 6.360179286 \\
\hline Hamming & 6.314117829 & 6.313367766 & 6.315545492 & 6.324429262 & 6.332322646 & 6.33409771 & 6.333856336 & 6.332585922 \\
\hline Blackman & 6.302260759 & 6.303272 & 6.303558226 & 6.308619677 & 6.312332078 & 6.31249273 & 41 & 6.312 \\
\hline Blackman-Harris & 6.318938009 & 6.317435825 & 6.32159714 & 38771007 & 31779 & 6.3616 & & \\
\hline Flat top & 6.317741146 & 6.316691593 & 6.320307479 & 6.336122551 & 6.352782751 & 6.356984761 & 6.354866599 & 6.35249844 \\
\hline Exponential & 6.320794905 & 6.318896804 & 6.323176328 & 6.339687335 & 6.3582 & 6.362522503 & 6.360466436 & 6.357282728 \\
\hline Gaussian & 6.3129 & 6.312397469 & 6.3143 & 6.3 & 6.329 & 6.3316 & 56 & \\
\hline Welch(Riesz) & 6.31298 & 6.312497347 & 6.31 & 6.323 & 6.329 & 6.331504406 & 6.331 & 6.330651116 \\
\hline Cosine & 6.317333361 & 6.316064139 & 6.319364383 & 6.332573978 & 6.345797365 & 6.349105366 & 6.347 & 6.345797365 \\
\hline Parzen & 6.321912789 & 6.319469585 & 6.323215615 & 6.336148995 & 6.354453504 & 6.356559699 & 6.3552 & 6.350618256 \\
\hline Bohman & 6.311609354 & 6.31128131 & 6.313085632 & 6.321097799 & 6.327607439 & 6.329162515 & 6.3290 & 6.328263199 \\
\hline Poisson & 6.310203987 & 6.309449206 & 6.311849782 & 6.321901239 & 6.331036428 & 6.333238834 & 6.332666646 & 6.331360452 \\
\hline Hanning-Poi & 6.308901111 & 6.308291579 & 6.310471547 & 6.319777571 & 6.32816172 & 6.330066936 & 6.329647765 & 6.328471139 \\
\hline Cauchy & 6.316534346 & 6.315404723 & 6.318452614 & 6.330768767 & 6.342655521 & 6.345688248 & 6.344625789 & 6.342838347 \\
\hline Bartlett-Hanning & 6.319845036 & 6.318180978 & 6.322379624 & 6.339105847 & 6.356579046 & 6.361152556 & 6.35903439 & 6.356414425 \\
\hline Min-MSE & Blackman & Blackman & Blackman & Blackman & Blackman & Blackman & Blackman & Blackman \\
\hline
\end{tabular}

TABLE 5: MSE values of MA(1) spectrum estimation with size $(n=100)$.

\begin{tabular}{|c|c|c|c|c|c|c|c|c|}
\hline \multirow{2}{*}{ Windows } & \multicolumn{8}{|c|}{ Different values of parameter $\beta$} \\
\hline & $\beta=-1.5$ & $\beta=-1$ & $\beta=-0.5$ & $\beta=0$ & $\beta=0.5$ & $\beta=1$ & $\beta=1.5$ & $\beta=2$ \\
\hline Rectangular & 6.317333361 & 6.316064139 & 6.319364383 & 6.332573978 & 6.345797365 & 6.349105366 & 6.347832956 & 6.345797365 \\
\hline Triangular & 6.316005269 & 6.315502259 & 6.316731003 & 6.321849529 & 6.326985356 & 6.327757368 & 6.326900064 & 6.32579965 \\
\hline Hanning & 6.31806519 & 6.316167753 & 6.32110453 & 6.341162052 & 6.360347991 & 6.365372888 & 6.364252615 & 6.360647922 \\
\hline Hamming & 6.316660153 & 6.315968816 & 6.317763862 & 6.324682905 & 6.33243793 & 6.334173767 & 6.332762578 & 6.332165359 \\
\hline Blackman & 6.315362421 & 6.315718152 & 6.314932087 & 6.310802524 & 6.308365932 & 6.307273013 & 6.306340167 & 6.306147794 \\
\hline Blackman-Harris & 6.317848489 & 6.316128283 & 6.320633953 & 6.33882696 & 6.356290341 & 6.360849096 & 6.359792368 & 6.356255191 \\
\hline Flat top & 6.317638588 & 6.316102605 & 6.320181441 & 6.336478466 & 6.352199786 & 6.356363117 & 6.355264552 & 6.351894477 \\
\hline Exponential & 6.318221266 & 6.31637155 & 6.321318053 & 6.340398962 & 6.360502172 & 6.36618394 & 6.364286426 & 6.36219272 \\
\hline Gaussian & 6.31645154 & 6.315884494 & 6.317326823 & 6.323030172 & 6.329212062 & 6.330404138 & 6.329199826 & 6.328365722 \\
\hline Welch(Riesz) & 6.316230368 & 6.315713366 & 6.316921366 & 6.32245267 & 6.327205341 & 6.327606375 & 6.326989738 & 6.325262877 \\
\hline Cosine & 6.317333361 & 6.316064139 & 6.319364383 & 6.332573978 & 6.345797365 & 6.349105366 & 6.347832956 & 6.345797365 \\
\hline Parzen & 6.319052677 & 6.317233201 & 6.322761625 & 6.339863334 & 6.366401923 & 6.374981893 & 6.370220836 & 6.373119197 \\
\hline Bohman & 6.316253855 & 6.315808587 & 6.316897146 & 6.321355062 & 6.325958709 & 6.326704444 & 6.325615407 & 6.324696778 \\
\hline Poisson & 6.315823722 & 6.315261275 & 6.31666959 & 6.322265743 & 6.328176627 & 6.329372137 & 6.328423389 & 6.327531799 \\
\hline Hanning-Poisson & 6.315661537 & 6.31523451 & 6.316291145 & 6.320398054 & 6.324977482 & 6.325848083 & 6.324882348 & 6.324291094 \\
\hline Cauchy & 6.317136032 & 6.31600932 & 6.318921608 & 6.330651747 & 6.342351301 & 6.345144417 & 6.343959584 & 6.342050158 \\
\hline Bartlett-Hanning & 6.317852285 & 6.316125822 & 6.320611356 & 6.338842477 & 6.356317622 & 6.36082472 & 6.359718723 & 6.356437047 \\
\hline Min-MSE & Blackman & Hanning-Poisson & Blackman & Blackman & Blackman & Blackman & Blackman & Blackman \\
\hline
\end{tabular}


TABLE 6: MSE values of MA(1) spectrum estimation with size $(n=500)$.

\begin{tabular}{|c|c|c|c|c|c|c|c|c|}
\hline \multirow{2}{*}{ Windows } & \multicolumn{8}{|c|}{ Different values of parameter $\beta$} \\
\hline & $\beta=-1.5$ & $\beta=-1$ & $\beta=-0.5$ & $\beta=0$ & $\beta=0.5$ & $\beta=1$ & $\beta=1.5$ & $\beta=2$ \\
\hline Rectangular & 6.317333361 & 6.316064139 & 6.319364383 & 6.332573978 & 6.345797365 & 6.349105366 & 6.347832956 & 6.345797365 \\
\hline Triangular & 6.316377185 & 6.315952991 & 6.317159619 & 6.321833153 & 6.326693206 & 6.326801285 & 6.326774568 & 6.325874867 \\
\hline Hanning & 6.317998059 & 6.316081423 & 6.32096733 & 6.340993146 & 6.360522531 & 6.365614361 & 6.363381808 & 6.361253929 \\
\hline Hamming & 6.3167219 & 6.316048238 & 6.317890033 & 6.324838119 & 6.332280487 & 6.333954984 & 6.333562764 & 6.331611969 \\
\hline Blackman & 6.315612827 & 6.316016966 & 6.31507097 & 6.311104415 & 6.307897643 & 6.306975267 & 6.306457259 & 6.30597069 \\
\hline Blackman-Harris & 6.317810644 & 6.3160 & 6.32049217 & 6.338673171 & 6.356415512 & 6.36107786 & 6.358807307 & 6986 \\
\hline Flat top & 6.317 & 57 & 6.320014315 & 6.336284248 & 6.352314562 & 6.35658088 & 6.354268933 & 6.35 \\
\hline Exponential & 6.318063049 & 6.316124968 & 6.321060167 & 6.340735216 & 6.360493164 & 6.367239265 & 6.364810109 & 6.361301206 \\
\hline Gaussian & 6.316557743 & 6.316032352 & 6.317501811 & 6.323066838 & 6.329037777 & 6.329918169 & 6.329657358 & 6.328171358 \\
\hline Welch(Riesz) & 6.316420872 & 6.315995647 & 6.31722662 & 6.322204748 & 6.32712537 & 6.3263227 & 6.326546164 & 6.326204822 \\
\hline Cosine & 6.317 & 6.3 & 64383 & 6.332573978 & 6.34 & 6.349105366 & 6.347832956 & 6.345797365 \\
\hline Parzen & 6.318577978 & 6.316312267 & 6.321970931 & 6.342973131 & 6.365195001 & 6.378587971 & 6.3755627 & 6.367369776 \\
\hline Bohman & 6.316393708 & 6.316018758 & 6.317117179 & 6.321211233 & 6.32579264 & 6.326101485 & 6.325881926 & 6.324644039 \\
\hline Poisson & 6.316 & 6.315 & 33642 & 6.322333304 & 6.327747006 & 6.328624662 & 6.328294354 & 6.327060016 \\
\hline Hanning-Poisson & 6.316239399 & 6.31590009 & 6.316876052 & 6.320464524 & 6.324529924 & 6.32515849 & 6.324897286 & 6.323651196 \\
\hline Cauchy & 6.317167665 & 6.316053652 & 6.318972459 & 6.330640244 & 6.342321031 & 6.344935342 & 6.343939081 & 6.342161726 \\
\hline Bartlett-H & 6.317810152 & 6.316073852 & 6.320517205 & 6.338685687 & 6.356453679 & 6.360945471 & 6.358981262 & 6.356983283 \\
\hline Min-MSE & Blackman & Hanning-Poisson & Blackman & Blackman & Blackman & Blackman & Blackman & Blackman \\
\hline
\end{tabular}

TABLE 7: MSE values of MA(1) spectrum estimation with size $(n=1000)$.

\begin{tabular}{|c|c|c|c|c|c|c|c|c|}
\hline \multirow{2}{*}{ Windows } & \multicolumn{8}{|c|}{ Different values of parameter $\beta$} \\
\hline & $\beta=$ & $\beta=-1$ & $\beta=-0.5$ & $\beta=0$ & $\beta=0.5$ & $\beta=1$ & $\beta=1.5$ & $\beta=2$ \\
\hline Rectangular & .317333361 & 16064139 & 6.319364383 & 6.332573978 & 6.345797365 & 6.349105366 & 6.347832956 & 6.345797365 \\
\hline Triangular & 316450439 & 6.316010799 & 6.317116444 & 6.320669904 & 6.326647222 & 6.327854445 & 6.326523968 & 6.326018604 \\
\hline Hanning & 17952234 & 6.316071633 & 6.320986333 & 6.341457537 & 6.360453565 & 6.364877187 & 6.364266231 & 6.360568627 \\
\hline Hamming & 6.316764056 & 6.316057244 & 6.317872553 & 6.324411712 & 6.332343093 & 6.334629512 & 6.332750509 & 6.332238824 \\
\hline Blackman & 6.315724711 & 6.316043998 & 6.314941086 & 6.309316698 & 6.307721597 & 6.307680769 & 6.307010748 & 6.307337395 \\
\hline Blackman-Harris & 6.317776777 & 6.316069431 & 6.320493511 & 6.338898231 & 6.356290763 & 6.360347095 & 6.35 & 56975 \\
\hline Flat top & 6093 & 342 & 6.320008079 & 6.33625915 & 6.352063455 & 6.35591282 & 6.355 & 7618 \\
\hline Exponential & 6.318033755 & 6.316091775 & 6.321147607 & 6.342219386 & 6.360665727 & 6.366052121 & 6.364741973 & 6.36159135 \\
\hline Gaussian & 6.316604308 & 6.316050059 & 6.317449541 & 6.322230584 & 6.329024221 & 6.330798748 & 6.329079574 & 6.328691584 \\
\hline Welch(Riesz) & 6.316455978 & 6.316033153 & 6.317132955 & 6.320433157 & 6.32697944 & 6.327870934 & 6.326603021 & 6.325936882 \\
\hline Cosine & 6.317 & 6.316064139 & 6.319364383 & 6.332573978 & 6.345797365 & 6.349105366 & 6.347832956 & 6.345797365 \\
\hline Parzen & 6.318549726 & 6.316178874 & 6.322383809 & 6.347943676 & 6.3665 & 6.376414843 & 6.37358532 & 6.370508696 \\
\hline Bohman & 6.316450929 & 6.316043932 & 6.317023713 & 6.32014857 & 6.325690506 & 6.327020438 & 6.325281865 & 6.325242589 \\
\hline Poisson & 6.316489926 & & 6.317228693 & 6.321646101 & 6.327736255 & 6.329177908 & 6.327932997 & 6.327379837 \\
\hline Hanning-Poisson & 6.31635782 & 6.315983781 & 6.31 & 6.319750927 & 6.324514567 & 6.325702868 & 6.324375171 & 6.324183754 \\
\hline Cauchy & 6.317174388 & 6.316059423 & 6.318959102 & 6.330351343 & 6.342309448 & 6.345216009 & 6.343919346 & 6.342150534 \\
\hline Bartlett-Hanning & 6.317775594 & 6.316068281 & 6.320526597 & 6.338945371 & 6.356382878 & 6.360441317 & 6.359704803 & 6.356411672 \\
\hline Min-MSE & Blackman & Hanning-Poisson & Blackman & Blackman & Blackman & Blackman & Blackman & Blackman \\
\hline
\end{tabular}

and the spectral density function $f\left(\omega_{i}\right)$ of $\mathrm{MA}(2)$ is given by

$$
f(\omega)=\frac{\left(1+\beta_{1}^{2}+\beta_{2}^{2}\right)+2\left(\beta_{1}+\beta_{1} \beta_{2}\right) \cos \omega+2 \beta_{2} \cos \omega}{2 \pi\left(1+\beta_{1}^{2}+\beta_{2}^{2}\right)}
$$$$
-\pi \leq \omega \leq \pi .
$$

(7) The criterion used to evaluate the windows performance was the mean square error (MSE) calculated with the following formula:

$$
\mathrm{MSE}=\frac{\sum_{j=1}^{k} \sum_{i=1}^{L}\left(f\left(\omega_{i}\right)-\widehat{f}_{j}\left(\omega_{i}\right)\right)^{2}}{k L},
$$

where $k$ and $L$ were defined in (3) and (5), respectively, and $\widehat{f}_{j}\left(\omega_{i}\right)$ is the consistent estimate of the SDF formula in (26). 
TABLE 8: MSE values of MA(1) spectrum estimation with size $(n=10000)$.

\begin{tabular}{|c|c|c|c|c|c|c|c|c|}
\hline \multirow{2}{*}{ Windows } & \multicolumn{8}{|c|}{ Different values of parameter $\beta$} \\
\hline & $\beta=-1.5$ & $\beta=-1$ & $\beta=-0.5$ & $\beta=0$ & $\beta=0.5$ & $\beta=1$ & $\beta=1.5$ & $\beta=2$ \\
\hline Rectangular & 6.317333361 & 6.316064139 & 6.319364383 & 6.332573978 & 6.345797365 & 6.349105366 & 6.347832956 & 6.345797365 \\
\hline Triangular & 6.316479573 & 6.31605864 & 6.317209379 & 6.321468334 & 6.325557514 & 6.326554021 & 6.325547978 & 6.325765604 \\
\hline Hanning & 6.317973878 & 6.316064925 & 6.321014701 & 6.341128648 & 6.361206169 & 6.365776987 & 6.363952022 & 6.360915895 \\
\hline Hamming & 6.316744145 & 6.316063416 & 6.31784647 & 6.324713492 & 6.331653522 & 6.333806476 & 6.333038184 & 6.331920613 \\
\hline Blackman & 6.315702546 & 6.316062088 & 6.315077573 & 6.310816877 & 6.305092725 & 6.305663759 & 6.305800605 & 6.308807089 \\
\hline Blackman-Harris & 6.317798694 & 6.316064702 & 6.320549488 & 6.338791963 & 6.356691063 & 6.360995072 & 6.359 & 2486 \\
\hline Flat top & 6.317 & 6.3160 & 6.320083994 & 6.336445166 & 6.352033339 & 6.356039601 & 6.354631114 & 6128 \\
\hline Exponential & 6.318011853 & 6.316067131 & 6.321018293 & 6.341142875 & 6.361633925 & 6.367540154 & 6.366462249 & 6.361999646 \\
\hline Gaussian & 6.316593257 & 6.316062641 & 6.317479185 & 6.322877081 & 6.328090783 & 6.329596079 & 6.328766781 & 6.32845892 \\
\hline Welch(Riesz) & 6.316481157 & & 6.317261818 & 6.321657877 & 6.325736418 & 6.326149294 & 6.324618196 & 6.32548475 \\
\hline Cosine & 6.317 & 6.316 & 64383 & 6.3325 & 365 & 6.349105366 & 6.347832956 & 6.345797365 \\
\hline Parzen & 6.318413973 & 6.316076684 & 6.321580321 & 6.34373885 & 6.368067039 & 6.381168828 & 6.381470473 & 6.371855429 \\
\hline Bohman & 6.316438799 & 6.316061985 & 6.31710822 & 6.321099464 & 6.32463446 & 6.32540767 & 6.324748977 & 6.32506562 \\
\hline Poisson & 6.316 & 6.316056095 & 6.317320073 & 6.322133862 & 6.3268 & 6.328248328 & 6.327603009 & 6.32730352 \\
\hline Hanning-Poisson & 6.316391338 & 6.31605599 & 6.316955154 & 6.320308685 & 6.323512583 & 6.32461211 & 6.324213674 & 6.324156156 \\
\hline Cauchy & 6.317177571 & 6.316063631 & 6.31897445 & 6.33054192 & 6.342080247 & 6.344927928 & 6.343628113 & 6.342071815 \\
\hline Bartlett-Ha & 6.317794749 & 6.316064567 & 6.320558417 & 6.338764354 & 6.356901826 & 6.361046061 & 6.359323892 & 6.356678845 \\
\hline Min-MSE & Blackman & Hanning-Poisson & Blackman & Blackman & Blackman & Blackman & Blackman & Blackman \\
\hline
\end{tabular}

TABLE 9: MSE values of MA(2) spectrum estimation with size $(n=10)$.

\begin{tabular}{|c|c|c|c|c|c|c|c|c|}
\hline \multirow[b]{2}{*}{ Windows } & \multicolumn{8}{|c|}{ Different values of parameters $\beta_{1}, \beta_{2}$} \\
\hline & $\begin{array}{c}\beta_{1}=1 \\
\beta_{2}=-1\end{array}$ & $\begin{array}{c}\beta_{1}=-1 \\
\beta_{2}=1\end{array}$ & $\begin{array}{l}\beta_{1}=0.5 \\
\beta_{2}=0.5\end{array}$ & $\begin{array}{l}\beta_{1}=-0.5 \\
\beta_{2}=-0.5\end{array}$ & $\begin{array}{c}\beta_{1}=0.5 \\
\beta_{2}=-0.5\end{array}$ & $\begin{array}{c}\beta_{1}=0.5 \\
\beta_{2}=1\end{array}$ & $\begin{array}{c}\beta_{1}=1 \\
\beta_{2}=-0.5\end{array}$ & $\begin{array}{c}\beta_{1}=-1.5 \\
\beta_{2}=1\end{array}$ \\
\hline Rectangular & 6.321565024 & 6.321565024 & 6.360138264 & 6.316064139 & 6.316064139 & 6.361978011 & .332573978 & 6.317034709 \\
\hline Triangular & 6.312005203 & 6.316707374 & 6.341600802 & 6.308265322 & 6.308265322 & 6.343799682 & 6.319994439 & 6.313156769 \\
\hline Hanning & 6.329331965 & 6.323962003 & 6.378387396 & 6.321932771 & 6.321932771 & 6.379542556 & 6.343610193 & 6.318259519 \\
\hline Hamming & 6.314426416 & 6.319360693 & 6.343378579 & 6.310669467 & 6.310669467 & 6.345847113 & 6.322433753 & 6.31590812 \\
\hline Blackman & 6.29540239 & 6.314673225 & 6.326490025 & 6.293526586 & 6.293526586 & 6.329993335 & 6.302467223 & 6.309973629 \\
\hline Blackman-Harris & 6.326158188 & 6.323331333 & 6.375330581 & 6.319121344 & 6.319121344 & 6.376639217 & 6.340221321 & 6.317448053 \\
\hline Flat top & 6.323428374 & 6.323339845 & 6.370649079 & 6.317007048 & 6.317007048 & 6.371981296 & 6.336830045 & 6.31758825 \\
\hline Exponential & 6.327426077 & 6.324458741 & 6.373850374 & 6.320445609 & 6.320445609 & 6.375765692 & 6.341101042 & 6.319028806 \\
\hline Gaussian & 6.31279587 & 6.318682021 & 6.341512875 & 6.309263029 & 6.309263029 & 6.343876486 & 6.320566376 & 6.315181164 \\
\hline Welch(Riesz) & 6.313917191 & 6.317934726 & 6.341881423 & 6.310367315 & 6.310367315 & 6.343773555 & 6.321380659 & 6.314643892 \\
\hline Cosine & 6.321565024 & 6.321565024 & 6.360138264 & 6.316064139 & 6.316064139 & 6.361978011 & 6.332573978 & 6.317034709 \\
\hline Parzen & 6.323495637 & 6.326494597 & 6.362753447 & 6.317361895 & 6.317361895 & 6.36755149 & 6.335711083 & 6.322006755 \\
\hline Bohman & 6.310834358 & 6.31799328 & 6.33935597 & 6.307546938 & 6.307546938 & 6.341615062 & 6.31841308 & 6.314405216 \\
\hline Poisson & 6.311294317 & 6.31569628 & 6.343823934 & 6.307123183 & 6.307123183 & 6.346107879 & 6.320193056 & 6.311748163 \\
\hline Hanning-Poisson & 6.309034828 & 6.314967826 & 6.34063026 & 6.30517467 & 6.30517467 & 6.343068984 & 6.317560801 & 6.311049725 \\
\hline Cauchy & 6.320072257 & 6.320909745 & 6.356521406 & 6.314953792 & 6.314953792 & 6.358409071 & 6.330385723 & 6.316629309 \\
\hline Bartlett-Hanning & 6.327290745 & 6.323273715 & 6.373709134 & 6.320385895 & 6.320385895 & 6.374995983 & 6.340729357 & 6.317872708 \\
\hline Min-MSE & Blackman & Blackman & Blackman & Blackman & Blackman & Blackman & Blackman & Blackman \\
\hline
\end{tabular}

\section{Results}

(1) For the first order of moving average process MA (1), when we study the different values of parameter $\beta$ as shown in Table 2 and size $n$ of the process, we get the results given in Tables $4-8$.

(2) For the second order of moving average process MA (2), when we study the different values of parameters $\beta_{1}, \beta_{2}$ and size of process $n$, we get the results given in Tables 9-13.

\section{Conclusion}

(1) In MA(1) with the different parameters and series sizes, the best lag window which gives the minimum mean square error (MSE) between the $\operatorname{SDF} f(\omega)$ and the consistent estimate of $\operatorname{SDF} \widehat{f}(\omega)$, and the results in Tables 4-8 can be summarized in Table 14.

(2) The results in Tables 9-13 shows that, in MA(2), with the following different parameters and series sizes, the best lag window which gives the minimum mean 
TABLE 10: MSE values of MA(2) spectrum estimation with size $(n=100)$.

\begin{tabular}{|c|c|c|c|c|c|c|c|c|}
\hline \multirow[b]{2}{*}{ Windows } & \multicolumn{8}{|c|}{ Different values of parameters $\beta_{1}, \beta_{2}$} \\
\hline & $\begin{array}{c}\beta_{1}=1 \\
\beta_{2}=-1\end{array}$ & $\begin{array}{c}\beta_{1}=-1 \\
\beta_{2}=1\end{array}$ & $\begin{array}{l}\beta_{1}=0.5 \\
\beta_{2}=0.5\end{array}$ & $\begin{array}{l}\beta_{1}=-0.5 \\
\beta_{2}=-0.5\end{array}$ & $\begin{array}{c}\beta_{1}=0.5 \\
\beta_{2}=-0.5\end{array}$ & $\begin{array}{c}\beta_{1}=0.5 \\
\beta_{2}=1\end{array}$ & $\begin{array}{c}\beta_{1}=1 \\
\beta_{2}=-0.5\end{array}$ & $\begin{array}{c}\beta_{1}=-1.5 \\
\beta_{2}=1\end{array}$ \\
\hline Rectangular & 6.321565024 & 6.321565024 & 6.360138264 & 6.316064139 & 6.327068304 & 6.361978011 & 6.332573978 & 6.317034709 \\
\hline Triangular & 6.317117423 & 6.31777068 & 6.333128008 & 6.315160112 & 6.31939826 & 6.333007724 & 6.321457949 & 6.316118821 \\
\hline Hanning & 6.324557448 & 6.324401497 & 6.382064674 & 6.316268444 & 6.332689354 & 6.385154783 & 6.340913819 & 6.317578586 \\
\hline Hamming & 6.3188133 & 6.318956624 & 6.34002707 & 6.315876185 & 6.32190157 & 6.34072313 & 6.324910813 & 6.316534387 \\
\hline Blackman & 6.313845839 & 6.314385509 & 6.304330089 & 6.315309193 & 6.312027333 & 6.301064093 & 6.310514309 & 6.315641361 \\
\hline Blackman-Harris & 6.323731576 & 6.323627073 & 6.376027133 & 6.316180189 & 6.331031122 & 6.378416063 & 6.338483312 & 6.317427316 \\
\hline Flat top & 6.322939163 & 6.322816414 & 6.369 & 6.316129535 & 6.325 & 6.371 & 6.336 & 6.317 \\
\hline Exponential & 6.324861824 & 6.324570285 & 6.380687038 & 6.316526045 & 6.33277471 & 6.384549625 & 6.341098715 & 6.317662753 \\
\hline Gaussian & 6.318063427 & 6.318296571 & 6.335634552 & 6.315744141 & 6.320580289 & 6.335698617 & 6.323003143 & 6.316391836 \\
\hline Welch(Riesz) & 6.317425293 & 6.317763848 & 6.333879433 & 6.315526842 & 6.319839881 & 6.333353187 & 6.321746436 & 6.316253592 \\
\hline Cosine & 6.321565024 & 6.321565024 & 6.360138264 & 6.316064139 & 6.327068304 & 6.361978011 & 6.332573978 & 6.317034709 \\
\hline Parzen & 6.327015561 & 6.326095813 & 6.383062251 & 6.31761612 & 6.335030668 & 6.39084977 & 6.344262279 & 6.318203885 \\
\hline Bohman & 6.317318887 & 6.317663081 & 6.33110916 & 6.315617866 & 6.319220643 & 6.33055528 & 6.321152307 & 6.316253023 \\
\hline Poisson & 6.317049932 & 6.318023383 & 6.334833716 & 6.314767193 & 6.319506318 & 6.335092961 & 6.32198115 & 6.316019062 \\
\hline Hanning-Poisson & 6.316388107 & 6.317432572 & 6.330017371 & 6.314701346 & 6.318221449 & 6.329926155 & 6.320154072 & 6.315904302 \\
\hline Cauchy & 6.320811057 & 6.320866313 & 6.355186428 & 6.315977384 & 6.325731496 & 6.356628158 & 6.330567105 & 6.316894163 \\
\hline Bartlett-Hanning & 6.323712379 & 6.323606229 & 6.376095466 & 6.316194872 & 6.331127061 & 6.378773405 & 6.338590688 & 6.317423083 \\
\hline Min-MSE & Blackman & Blackman & Blackman & Hanning-Poisson & Blackman & Blackman & Blackman & Blackman \\
\hline
\end{tabular}

TABLE 11: MSE values of MA(2) spectrum estimation with size $(n=500)$.

\begin{tabular}{|c|c|c|c|c|c|c|c|c|}
\hline \multirow[b]{2}{*}{ Windows } & \multicolumn{8}{|c|}{ Different values of parameters $\beta_{1}, \beta_{2}$} \\
\hline & $\begin{array}{c}\beta_{1}=1 \\
\beta_{2}=-1\end{array}$ & $\begin{array}{c}\beta_{1}=-1 \\
\beta_{2}=1\end{array}$ & $\begin{array}{l}\beta_{1}=0.5 \\
\beta_{2}=0.5\end{array}$ & $\begin{array}{l}\beta_{1}=-0.5 \\
\beta_{2}=-0.5\end{array}$ & $\begin{array}{c}\beta_{1}=0.5 \\
\beta_{2}=-0.5\end{array}$ & $\begin{array}{c}\beta_{1}=0.5 \\
\beta_{2}=1\end{array}$ & $\begin{array}{c}\beta_{1}=1 \\
\beta_{2}=-0.5\end{array}$ & $\begin{array}{c}\beta_{1}=-1.5 \\
\beta_{2}=1\end{array}$ \\
\hline Rectangular & 6.321565024 & 6.321565024 & 6.360138264 & 6.316064139 & 6.327068304 & 6.361978011 & 6.332573978 & 6.317034709 \\
\hline Triangular & 6.317647266 & 6.3179566 & 6.331517324 & 6.31588179 & 6.319328483 & 6.332050539 & 6.321301252 & 6.31 \\
\hline Hanning & 6.324250822 & 6.324265461 & 6.38206513 & 6.316091945 & 6.332703845 & 6.384872656 & 6.340870645 & 6.317506423 \\
\hline Hamming & 6.31909505 & 6.319081614 & 6.340031293 & 6.316038557 & 6.321887866 & 6.340985719 & 6.324950371 & 6.316600764 \\
\hline Blackman & 31455688 & 6.314500 & 6.30225 & 6.315 & 8132 & 6.30191 & 6.3109 & 6.315 \\
\hline Blackman-Harris & 6.32348736 & 6.323494163 & 6.375675873 & 6.3160 & 988 & 6.378262669 & 6.338 & 6.317 \\
\hline Flat top & 6.322718912 & 6.322716438 & 6.369293183 & 6.31607 & 7997 & 6.371648059 & 6.3362 & 6.317227254 \\
\hline Exponential & 6.324840245 & 6.324407841 & 6.382496376 & 6.316163699 & 49526 & 6.385428999 & 6.341664876 & 6.317 \\
\hline Gaus & 74 & 59 & 6.3 & & 191 & 6.33 & 6.322 & 275 \\
\hline Welch(Riesz) & 6.317505476 & 6.31797286 & 6.3319 & 6.3 & 721 & 6.332 & 6.321 & 6.31 \\
\hline Cosine & 6.321565024 & 6.321565024 & 6.360138264 & & 6.327068304 & 6.361978011 & 6.33257 & 6.3170 \\
\hline Parzen & 6.328047906 & 6.325832428 & 6.392371593 & 6.316475625 & 6.337532096 & 6.395529955 & 6.347464105 & 6.317834331 \\
\hline Bohman & 6.317608082 & 6.317812419 & 6.3297 & 6.31 & 591 & 6.330 & 6.3209 & 4675 \\
\hline Poisson & 6.317936785 & 6.318181261 & 6.3331846 & 6.3157986 & 6772 & 6.333917098 & 6.322115039 & 6.316370761 \\
\hline Hanning-Poisson & 6.317367925 & 6.317591571 & 6.328278066 & 6.31579334 & 6.318733654 & 6.328904817 & 6.320328848 & 6.316263887 \\
\hline Cauchy & 6.320839839 & 6.320904802 & 6.354921707 & 6.316047154 & 6.325630499 & 6.356491956 & 6.330489775 & 6.31691898 \\
\hline Bartlett-Hanning & 6.323469349 & 6.32350886 & 6.375966284 & 6.316079627 & 6.331101469 & 6.378496766 & 6.338527326 & 6.317373725 \\
\hline Min-MSE & Blackman & Blackman & Blackman & Hanning-Poisson & Blackman & Blackman & Blackman & Blackman \\
\hline
\end{tabular}

square error (MSE) between the SDF $f(\omega)$ and the consistent estimate of $\operatorname{SDF} \widehat{f}(\omega)$ is shown in Table 15.

(3) In MA (1) with series sizes $n<40$ and any value of parameter $\beta$, the best lag window which gives the minimum mean square error (MSE) between the $\operatorname{SDF} f(\omega)$ and the consistent estimate of $\operatorname{SDF} \widehat{f}(\omega)$ is the Blackman window, as shown in Table 9.

(4) In MA(1) with series sizes $n \geq 40$ and parameter $\beta=-1$ or belongs to neighborhood -1 with radius
0.3 , the best lag window which gives the minimum mean square error (MSE) between the SDF $f(\omega)$ and the consistent estimate of $\operatorname{SDF} \hat{f}(\omega)$ is the Hanning-Poisson window, as shown in Table 9.

(5) Blackman window is the best window to estimate the SDF for white noise $\epsilon_{t}$, where $\operatorname{MA}(1)=\epsilon_{t}$ when $\beta=0$, as shown in Table 9.

(6) In MA (2) with series sizes $n<40$ and any values of parameters $\beta_{1}, \beta_{2}$, the best lag window which gives the minimum mean square error (MSE) between the 
TABLE 12: MSE values of MA(2) spectrum estimation with size $(n=1000)$.

\begin{tabular}{|c|c|c|c|c|c|c|c|c|}
\hline \multirow[b]{2}{*}{ Windows } & \multicolumn{8}{|c|}{ Different values of parameters $\beta_{1}, \beta_{2}$} \\
\hline & $\begin{array}{c}\beta_{1}=1 \\
\beta_{2}=-1\end{array}$ & $\begin{array}{c}\beta_{1}=-1 \\
\beta_{2}=1\end{array}$ & $\begin{array}{l}\beta_{1}=0.5 \\
\beta_{2}=0.5\end{array}$ & $\begin{array}{l}\beta_{1}=-0.5 \\
\beta_{2}=-0.5\end{array}$ & $\begin{array}{c}\beta_{1}=0.5 \\
\beta_{2}=-0.5\end{array}$ & $\begin{array}{c}\beta_{1}=0.5 \\
\beta_{2}=1\end{array}$ & $\begin{array}{c}\beta_{1}=1 \\
\beta_{2}=-0.5\end{array}$ & $\begin{array}{c}\beta_{1}=-1.5 \\
\beta_{2}=1\end{array}$ \\
\hline Rectangular & 6.321565024 & 6.321565024 & 6.360138264 & 6.316064139 & 6.327068304 & 6.361978011 & 6.332573978 & 6.317034709 \\
\hline Triangular & 6.317820977 & 6.317924846 & 6.330318905 & 6.315974265 & 6.319357377 & 6.331935269 & 6.322220406 & 6.31635336 \\
\hline Hanning & 6.324327739 & 6.324326234 & 6.382575142 & 6.316078585 & 6.332692347 & 6.385185403 & 6.340355037 & 6.317535755 \\
\hline Hamming & 6.319024374 & 6.319025792 & 6.339567906 & 6.316050848 & 6.321898419 & 6.340702612 & 6.325423809 & 6.316573783 \\
\hline Blackman & 6.314648712 & 6.314272258 & 6.301303387 & 6.31602455 & 6.312563425 & 6.303054634 & 6.311731336 & 6.315768701 \\
\hline Blackman-Harris & 6.323590394 & 6.323528609 & 6.376110223 & 6.316074223 & 6.331115503 & 6.378782602 & 6.33805233 & 6.317400392 \\
\hline Flat top & 6.322829702 & 6.32274256 & 6.369677188 & 6.316 & 9258 & 6.3721 & 6.335 & 6.31 \\
\hline Exponential & 6.324558834 & 6.324377777 & 6.38375854 & 6.316109209 & 6.333391536 & 6.385552536 & 6.340534867 & 6.317564817 \\
\hline Gaussian & 6.318372808 & 6.318392146 & 6.334241625 & 6.316038988 & 6.320469553 & 6.335580791 & 6.323583399 & 6.316457184 \\
\hline Welch(Riesz) & 6.317801343 & 6.318001235 & 6.330356383 & 6.316012613 & 6.319149002 & 6.33221923 & 6.322532917 & 6.316363813 \\
\hline Cosine & 6.321565024 & 6.321565024 & 6.360138264 & 6.316064139 & 6.327068304 & 6.361978011 & 6.332573978 & 6.317034709 \\
\hline Parzen & 6.32641214 & 6.325521793 & 6.39535827 & 6.316237555 & 6.337953601 & 6.395432172 & 6.344184482 & 6.317829935 \\
\hline Bohman & 6.31773952 & 6.317754417 & 6.329118219 & 6.316029039 & 6.319103227 & 6.330499792 & 6.321678537 & 6.316343762 \\
\hline Poisson & 6.318063244 & 6.31811306 & 6.332348143 & 6.315931055 & 6.319983864 & 6.33388695 & 6.322647099 & 6.316386733 \\
\hline Hanning-Poisson & 6.317481915 & 6.317495665 & 6.32750183 & 6.315928748 & 6.318794459 & 6.328913444 & 6.320862533 & 6.316280627 \\
\hline Cauchy & 6.320877617 & 6.320906541 & 6.354635902 & 6.316056093 & 6.325630084 & 6.356453375 & 6.330722247 & 6.316911431 \\
\hline Bartlett-Hanning & 6.323551102 & 6.323559592 & 6.376281513 & 6.316072595 & 6.331091791 & 6.378745441 & 6.338191821 & 6.317395507 \\
\hline Min-MSE & Blackman & Blackman & Blackman & Hanning-Poisson & Blackman & Blackman & Blackman & Blackman \\
\hline
\end{tabular}

TABLE 13: MSE values of MA(2) spectrum estimation with size $(n=10000)$.

\begin{tabular}{|c|c|c|c|c|c|c|c|c|}
\hline \multirow[b]{2}{*}{ Windows } & \multicolumn{8}{|c|}{ Different values of parameters $\beta_{1}, \beta_{2}$} \\
\hline & $\begin{array}{c}\beta_{1}=1 \\
\beta_{2}=-1\end{array}$ & $\begin{array}{c}\beta_{1}=-1 \\
\beta_{2}=1\end{array}$ & $\begin{array}{l}\beta_{1}=0.5 \\
\beta_{2}=0.5\end{array}$ & $\begin{array}{l}\beta_{1}=-0.5 \\
\beta_{2}=-0.5\end{array}$ & $\begin{array}{c}\beta_{1}=0.5 \\
\beta_{2}=-0.5\end{array}$ & $\begin{array}{c}\beta_{1}=0.5 \\
\beta_{2}=1\end{array}$ & $\begin{array}{c}\beta_{1}=1 \\
\beta_{2}=-0.5\end{array}$ & $\begin{array}{c}\beta_{1}=-1.5 \\
\beta_{2}=1\end{array}$ \\
\hline Rectangular & 6.321565024 & 6.321565024 & 6.360138264 & 6.316064139 & 6.327068304 & 6.361978011 & 6.332573978 & 6.317034709 \\
\hline Triangular & 6.317855079 & 6.318048015 & 6.329728119 & 6.31605515 & 6.319269444 & 6.331734076 & 6.321390891 & 6.31638888 \\
\hline Hanning & 6.324333631 & 6.324210633 & 6.383194947 & 6.316065401 & 6.33274672 & 6.38583431 & 6.340955416 & 6.317515983 \\
\hline Hamming & 6.319018997 & 6.319132064 & 6.338999008 & 6.316062978 & 6.321848752 & 6.34011287 & 6.324872728 & 6.316591972 \\
\hline Blackman & 6.314602201 & 6.314606383 & 6.302553934 & 6.316060833 & 6.31262263 & 6.304153737 & 6.311101155 & 6.315776292 \\
\hline Blackman-Harris & 6.323590977 & 6.323453625 & 6.376995885 & 6.316065041 & 6.331187991 & 6.379739984 & 6.338630758 & 6.317378991 \\
\hline Flat top & 6.32283238 & 6.322718592 & 6.370476692 & 6.31606468 & 6.329578574 & 6.373369557 & 6.33625461 & 6.317242264 \\
\hline Exponential & 6.324542244 & 6.324301408 & 6.38464717 & 6.316068875 & 6.333565517 & 6.385292434 & 6.341509798 & 6.317 \\
\hline Gaussian & 6.318369328 & 6.31851271 & 6.333705322 & 6.316061752 & 6.320389388 & 6.335275274 & 6.322893501 & 6.316477891 \\
\hline Welch(Riesz) & 6.317807718 & 6.318110539 & 6.329288881 & 6.316058835 & 6.318937909 & 6.332372703 & 6.321295763 & 6.316390903 \\
\hline Cosine & 6.321565024 & 6.321565024 & 6.360138264 & 6.316064139 & 6.327068304 & 6.361978011 & 6.332573978 & 6.317034709 \\
\hline Parzen & 6.326203496 & 6.325611467 & 6.39781678 & 6.316083922 & 6.338511857 & 6.391717309 & 6.346256854 & 6.317781749 \\
\hline Bohman & 6.317746178 & 6.317883746 & 6.328662769 & 6.316060713 & 6.319016136 & 6.33040712 & 6.320991031 & 6.31636812 \\
\hline Poisson & 6.318134832 & 6.318228945 & 6.332174728 & 6.316050845 & 6.320001297 & 6.33355514 & 6.322214269 & 6.316430102 \\
\hline Hanning-Poisson & 6.317554127 & 6.317635162 & 6.327408815 & 6.316050674 & 6.318825202 & 6.328555408 & 6.320436569 & 6.316326227 \\
\hline Cauchy & 6.320876447 & 6.320929331 & 6.354453735 & 6.316063333 & 6.325595081 & 6.356433889 & 6.33050151 & 6.316916254 \\
\hline Bartlett-Hanning & 6.323555462 & 6.323474752 & 6.376724998 & 6.316064831 & 6.331122539 & 6.379295097 & 6.338600735 & 6.31738112 \\
\hline Min-MSE & Blackman & Blackman & Blackman & Hanning-Poisson & Blackman & Blackman & Blackman & Blackman \\
\hline
\end{tabular}

TABLE 14: Best lag window with minimum MSE between $f(\omega)$ and $\hat{f}(\omega)$. of MA (1).

\begin{tabular}{|c|c|c|c|c|c|c|c|c|}
\hline \multirow{2}{*}{ Sample size } & \multicolumn{8}{|c|}{ Parameters } \\
\hline & $\beta=-1.5$ & $\beta=-1$ & $\beta=-0.5$ & $\beta=0$ & $\beta=0.5$ & $\beta=1$ & $\beta=1.5$ & $\beta=2$ \\
\hline 10 & Blackman & Blackman & Blackman & Blackman & Blackman & Blackman & Blackman & Blackman \\
\hline 100 & Blackman & Hanning-Poisson & Blackman & Blackman & Blackman & Blackman & Blackman & Blackman \\
\hline 500 & Blackman & Hanning-Poisson & Blackman & Blackman & Blackman & Blackman & Blackman & Blackman \\
\hline 1000 & Blackman & Hanning-Poisson & Blackman & Blackman & Blackman & Blackman & Blackman & Blackman \\
\hline 10000 & Blackman & Hanning-Poisson & Blackman & Blackman & Blackman & Blackman & Blackman & Blackman \\
\hline
\end{tabular}


TABLE 15: Best lag window with minimum MSE between $f(\omega)$ and $\hat{f}(\omega)$ of.MA(1)

\begin{tabular}{|c|c|c|c|c|c|c|c|c|}
\hline \multirow[b]{2}{*}{ Series size $n$} & \multicolumn{8}{|c|}{ Parameters } \\
\hline & $\begin{array}{c}\beta_{1}=1 \\
\beta_{2}=-1\end{array}$ & $\begin{array}{c}\beta_{1}=-1 \\
\beta_{2}=1\end{array}$ & $\begin{array}{l}\beta_{1}=0.5 \\
\beta_{2}=0.5\end{array}$ & $\begin{array}{l}\beta_{1}=-0.5 \\
\beta_{2}=-0.5\end{array}$ & $\begin{array}{c}\beta_{1}=0.5 \\
\beta_{2}=-0.5\end{array}$ & $\begin{array}{c}\beta_{1}=0.5 \\
\beta_{2}=1\end{array}$ & $\begin{array}{c}\beta_{1}=1 \\
\beta_{2}=-0.5\end{array}$ & $\begin{array}{c}\beta_{1}=-1.5 \\
\beta_{2}=1\end{array}$ \\
\hline 10 & Blackman & Blackman & Blackman & Blackman & Blackman & Blackman & Blackman & Blackman \\
\hline 100 & Blackman & Blackman & Blackman & Hanning-Poisson & Blackman & Blackman & Blackman & Blackman \\
\hline 500 & Blackman & Blackman & Blackman & Hanning-Poisson & Blackman & Blackman & Blackman & Blackman \\
\hline 1000 & Blackman & Blackman & Blackman & Hanning-Poisson & Blackman & Blackman & Blackman & Blackman \\
\hline 10000 & Blackman & Blackman & Blackman & Hanning-Poisson & Blackman & Blackman & Blackman & Blackman \\
\hline
\end{tabular}

$\operatorname{SDF} f(\omega)$ and the consistent estimate of $\operatorname{SDF} \hat{f}(\omega)$ is the Blackman window, as shown in Table 15.

(7) In MA(2) with series sizes $n \geq 40$ and parameters $\beta_{1}=\beta_{2}=-0.5$, the best lag window is the Hanning-Poisson window, or the Blackman window, as shown in Table 15.

\section{Data Availability}

The data included in the article were calculated using the MATLAB software and the method of calculation of the data is given in Section 6.

\section{Conflicts of Interest}

The authors declare that they have no conflicts of interest.

\section{References}

[1] H. Harris, Applied Time Series Modelling and Forecast, John Wiley \& Sons, Hoboken, NJ, USA, 2003.

[2] W. Enders, Applied Econometric Time Series, John Wiley \& Sons, New York, NY, USA, 1995.

[3] M. Priestley, Spectral Analysis and Time Series, Academic Press, London, UK, 1981.

[4] S. Ng and P. Perron, "The exact error in estimating the spectral density at the origin," Journal of Time Series Analysis, vol. 17, no. 4, pp. 379-408, 1996.

[5] E. Turkbeyler, "A three dimensional window function for the consistent estimation of the trispectrum," in Proceedings of the First Balkan Conference on Signal Processing, Communications, Circuits, and Systems, Istanbul, Turkey, January 2000.

[6] C. Zhongsheng, Y. Yongmin, and W. Xisen, "Improvement of bispectrum estimation based on one new 2-D combined lag window," in Proceedings of the International Conference on Robotics, Intelligent Systems and Signal Processing, IEEE, Changsha, China, October 2003.

[7] L. Zhang, K. Chen, and M. Pan, "Spectral methods in spatial statistics," Discrete Dynamics in Nature and Society, vol. 2014, Article ID 380392, 12 pages, 2014.

[8] T. Subba, "Developments in time series analysis," Champman and Hall\CRC, Boca Raton, FL, USA, 1993.

[9] G. M. Jenkins and D. G. Watts, Spectral Analysis and Its Applications. in Holden Day, Cambridge University Press, Cambridge, UK, 1968.

[10] G. Chen, B. Abraham, and S. Peiris, "Lag window estimation of the degree of differencing in fractionally integrated time series models," Journal of Time Series Analysis, vol. 15, no. 5, pp. 473-487, 1994.
[11] F. J. Harris, "On the use of windows for harmonic analysis with the discrete Fourier transform," Proceedings of the IEEE, vol. 66, no. 1, pp. 51-83, 1978.

[12] A. H. Nuttall, "Some windows with very good sidelobe behavior," IEEE Transactions on Acoustics, Speech, and Signal Processing, vol. 29, no. 1, 1981. 